\section{5-S4.02 SUCCESSFUL INTERVENTIONS TO INCREASE USE OF PATIENT SELF OBTAINED VAGINAL SWABS FOR CHLAMYDIA/GONORRHOEA TESTING IN WA STATE}

doi:10.1136/sextrans-2011-050109.170

${ }^{1} \mathrm{~S}$ Goldenkranz, ${ }^{1} \mathrm{D}$ Fine, ${ }^{2} \mathrm{C}$ Knutson, ${ }^{2} \mathrm{R}$ Loza. ${ }^{1}$ Center for Health Training Seattle, USA; ${ }^{2}$ Washington State Department of Health, USA

Background Patient Self-Obtained Vaginal Swabs (SOVs) are the most sensitive and specific technology for chlamydia (CT) and gonorrhoea (GC) testing. While SOVs enhance clinic efficiency and client access to testing, uptake of this innovation has been slow. The 10 USPHS Regional Infertility Prevention Projects (IPP) collect CT/GC test data from Family Planning, STD, and other publicly-funded clinics across the U.S. By 2010, only two regional IPPs reported using vaginal swabs. Washington (WA) State has been a leader in SOV promotion and collects common supplemental data to evaluate uptake.

Objectives (1) Describe WA SOV interventions; (2) Describe clinics' adoption and use of SOVs; (3) Explore trends and associations in SOV use vs other specimen sources.

Methods In 2011, key stakeholders were interviewed about SOV interventions. WA IPP data from female patients were compiled from $1 / 2007$ to $6 / 2010$. Clinic and client factors associated with SOVs were explored including visit date, clinic type, client demographics, specimen source, and test results. Multivariate models were developed. An aggregate clinic-level dataset was generated to assess SOV uptake in clinics averaging $>25$ tests/year. Significant $(\mathrm{p}<0.05)$ results are reported.

Results In 2008, WA Department of Health declared SOV promotion a state-wide objective. Interventions included development and dissemination of patient instructions, provider education materials and webinars; direct communication and follow-up with clinic management; and data monitoring. Of 126 IPP clinics, \% using SOVs increased significantly from $9.3 \%$ in 2007 to $76.8 \%$ in 2010. Among clinics using SOVs, mean \% of CT/GC specimen obtained by SOVs increased from $1.7 \%$ to $27.2 \%$. During the study period, 176,234 total CT/GC specimen were obtained. SOV use rose $16.6 \%$ overall (from $0.3 \%$ in 2007 to $16.9 \%$ in 2010 ), replacing $12.2 \%$ of clinician-obtained and $4.4 \%$ of urine-based specimen (Abstract O5S4.02 table 1). Factors independently associated with SOV use were: client age $<20 \quad(\mathrm{AOR}=1.55)$ and clinic type (FP: ref., STD: $\mathrm{AOR}=0.27$, School-Based: $\mathrm{AOR}=5.44$, Women's Health: $\mathrm{AOR}=0.07$ ). Client race, ethnicity, and CT/GC positivity were not associated.

Abstract 05-S4.02 Table 1 Chlamydia/Gonorrhea specimen source distribution by year (CT/GC test data)

\begin{tabular}{|c|c|c|c|c|c|c|c|c|c|}
\hline & \multicolumn{2}{|l|}{2007} & \multicolumn{2}{|l|}{$2008 \dagger$} & \multicolumn{2}{|l|}{2009} & \multicolumn{2}{|l|}{2010} & \multirow{2}{*}{$\begin{array}{l}\% \text { change, } \\
2007-2010\end{array}$} \\
\hline & $\mathrm{N}$ & $\%$ & $\mathrm{~N}$ & $\%$ & $\mathbf{N}$ & $\%$ & $\mathbf{N}$ & $\%$ & \\
\hline $\begin{array}{l}\text { Clinican- } \\
\text { Obtained }^{*}\end{array}$ & 25076 & $52.0 \%$ & 23231 & $45.3 \%$ & 22814 & $42.2 \%$ & 8885 & $39.8 \%$ & $-12.2 \%$ \\
\hline Urine & 23024 & $47.7 \%$ & 26785 & $52.2 \%$ & 25257 & $46.7 \%$ & 9677 & $43.4 \%$ & $-4.4 \%$ \\
\hline SOV & 123 & $0.3 \%$ & 1290 & $2.5 \%$ & 5984 & $11.1 \%$ & 3743 & $16.8 \%$ & $16.6 \%$ \\
\hline Total & 48223 & $100.0 \%$ & 51306 & $100.0 \%$ & 54055 & $100.0 \%$ & 22305 & $100.0 \%$ & $0.0 \%$ \\
\hline
\end{tabular}

${ }^{*}$ Clinician-obtained method include endocervical and clinician -obtained vaginal swabs. +SOV promotion intervention initiated in 2008. $\chi^{2}<0.001$.

Conclusions Since 2007, SOV use increased substantially across WA state and within individual clinics. SOVs are replacing clinicianobtained and urine specimens. More work is needed to improve uptake across the variety of clinic types. WA's successful intervention and evaluation activities can be a model for other states.

\section{5-S4.03 HIV TESTING AMONG PATIENTS INFECTED WITH NEISSERIA GONORRHOEAE-STD SURVEILLANCE NETWORK, USA, 2009-2010}

doi:10.1136/sextrans-2011-050109.171

${ }^{1} \mathrm{H}$ Bradley, ${ }^{2} \mathrm{~L}$ Asbel, ${ }^{3} \mathrm{~K}$ Bernstein, ${ }^{4} \mathrm{M}$ Mattson, ${ }^{5} \mathrm{P}$ Pathela, ${ }^{6} \mathrm{R}$ Pino, ${ }^{7} \mathrm{M}$ Samuel, ${ }^{8} \mathrm{~J}$ Schwebke, ${ }^{9} \mathrm{M}$ Stenger, ${ }^{10} \mathrm{~T}$ Tabidze, ${ }^{11} \mathrm{~J}$ Zenilman, ${ }^{1} \mathrm{D}$ Dowell, ${ }^{1} \mathrm{H}$ Weinstock. ${ }^{1}$ Centers for Disease Control and Prevention, Atlanta, USA; ${ }^{2}$ Philadelphia Department of Public Health, USA; ${ }^{3}$ San Francisco Department of Public Health, USA; ${ }^{4}$ Colorado Department of Public Health and Environment, USA; ${ }^{5}$ Department of Health and Mental Hygiene, New York, USA; ${ }^{6}$ Department of Health, Connecticut, USA; ${ }^{7}$ Department of Public Health, California, USA; ${ }^{8}$ University of Alabama, Birmingham, USA; ${ }^{9}$ Department of Health, Washington, USA; ${ }^{10}$ Department of Public Health, Chicago, USA; ${ }^{11}$ Johns Hopkins University, School of Medicine, USA

Background An estimated 21\% of 1.1 million HIV-infected US residents do not know they are infected and may unknowingly transmit HIV. Because people with sexually transmitted diseases (STDs) are at increased risk of HIV, CDC recommends HIV screening for patients seeking STD treatment. We investigated whether patients given a diagnosis of Neisseria gonorrhoeae during January 2009-June 2010 were concurrently tested for HIV.

Methods We used data from interviews with randomly selected gonorrhoea-infected patients in the 12 state and local health jurisdictions constituting the STD Surveillance Network. We compared the prevalence of HIV testing concurrent with gonorrhoea testing or treatment among patients seeking care in STD clinics and patients in other practice settings (emergency rooms, public and private outpatient facilities, hospitals, and family planning clinics).

Results Of 6658 eligible patients, 3462 (52\%) were successfully interviewed. Complete data were available for 1845/3462 (53\%). Of these, $51 \%$ were tested for HIV when they were tested or treated for gonorrhoea. The proportion of gonorrhoea patients tested for HIV ranged from $22 \%$ to $74 \%$ by jurisdiction. STD clinic patients were more likely to be tested for HIV than those in other practice settings (61\% vs 46\%, p <0.01), see Abstract O5-S4.03 figure 1 and women were more likely than men to be tested for HIV in STD clinics (71\% vs $58 \%, p=0.01$ ). HIV testing was more common among black $(53 \%)$ and Hispanic women $(54 \%)$ compared with white women $(36 \%, p=0.01)$. Men who have sex with men (MSM) were generally more likely to be tested for HIV compared with other men (56\% vs $48 \%, p=0.02$ ), though $58 \%$ of both MSM and MSW were tested for HIV in STD clinics. We found no association between HIV testing and race (among men), age, or having multiple sex partners.

Conclusions HIV testing among gonorrhoea-infected patients is suboptimal. While patients are more likely to be HIV-tested in STD clinics than in other practice settings, even STD clinics miss many

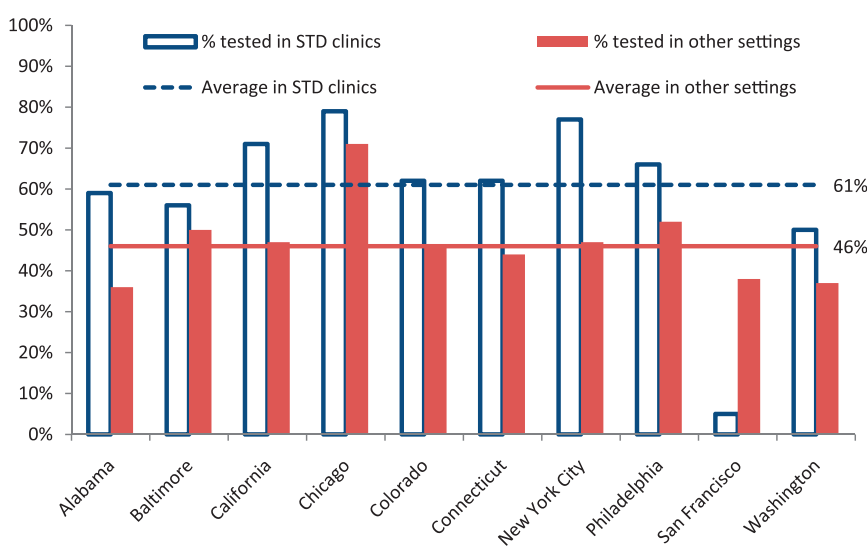

Abstract 05-S4.03 Figure 1 Percentage of patients tested for HIV concurrently with gonorrhea testing or treatment by facility type and jurisdiction. 
opportunities to test high risk groups such as MSM, who are less likely than women to be HIV tested in this setting. Interventions are urgently needed to increase HIV testing among STD patients.

\section{5-S4.04 TARGETED STD SCREENING AMONG HIGH-RISK MEN WHO HAVE SEX WITH MEN}

doi:10.1136/sextrans-2011-050109.172

R Perkins, B Furness, M Hager, R McLaughlin, J Goforth, M Davies. Whitman-Walker Clinic, Washington, USA

Background Subgroups of men who have sex with men (MSM) are at high risk for HIV infection and other viral and bacterial STDs. Since the mid-1990s, increased rates of early syphilis, gonorrhoea, and chlamydial infection and higher rates of unsafe sexual behaviours have been documented among MSM in the USA and virtually all industrialised countries.

Methods The Gay Men's Health \& Wellness (GMH\&W) Clinic is a Tuesday and Thursday evening STD clinic for men at WhitmanWalker Clinic (WWC), a Washington, DC community health center specialising in HIV/AIDS care and lesbian, gay, bisexual, and transgender care. As an outreach of this bi-weekly clinic, WWC, Gilead Foundation, and the local HIV/AIDS, Hepatitis, STD, and TB Administration collaborated to provide STD screening in the Crew Club, the District's only bathhouse serving MSM, for $\sim 4 \mathrm{~h}$ each Tuesday night beginning July 2010. All participating men were offered HIV screening, syphilis screening, and nucleic acid amplification testing NAAT) for gonorrhoea and chlamydia of urine, pharyngeal, and rectal specimens.

Results During the first 6 months of testing at the Crew Club, 173 unique clients were screened. Of those, five new HIV and 18 new syphilis cases were identified (Abstract O5-S4.04 table 1). In addition, two urethral, six pharyngeal, and two rectal gonorrhoeal infections were identified and five urethral, two pharyngeal, and 14 rectal chlamydial infections were identified. In comparison, during 2010, 2487 unique clients were tested at the GMH\&W Clinic. Of those, $37 \mathrm{HIV}$ and 189 syphilis cases were identified. In addition, 115 urethral and 89 rectal gonorrhoeal infections were identified and 139 urethral and 124 rectal chlamydial infections were identified. Pharyngeal results from the GMH\&W Clinic were not yet available.

Abstract 05-\$4.04 Table 1 Testing results, gay men's health \& wellness clinic (2010) vs STD Screening at the Crew Club (July-December 2010)

\begin{tabular}{llllllll}
\hline & \multicolumn{3}{l}{ GMH\&W clinic } & \multicolumn{3}{l}{ Crew club } \\
\cline { 2 - 3 } Test & Tests & Positives & \% Positive & & Tests & Positives & \% Positive \\
\hline HIV & 2747 & 37 & $1.35 \%$ & & 189 & 7 & $3.70 \%$ \\
Syphilis & 2969 & 189 & $6.37 \%$ & & 191 & 18 & $9.42 \%$ \\
Urethral GC & 1753 & 115 & $6.56 \%$ & & 184 & 2 & $1.09 \%$ \\
Pharyngeal GC & 2017 & N/A & - & & 189 & 6 & $3.17 \%$ \\
Rectal GC & 1138 & 89 & $7.82 \%$ & & 183 & 2 & $1.09 \%$ \\
Urethral CT & 1753 & 139 & $7.93 \%$ & & 184 & 5 & $2.72 \%$ \\
Pharyngeal CT & 2017 & N/A & - & & 189 & 2 & $1.06 \%$ \\
Rectal CT & 1138 & 124 & $10.90 \%$ & & 183 & 14 & $7.65 \%$ \\
\hline
\end{tabular}

Conclusions A higher percentage of presumably asymptomatic clients tested positive for HIV and syphilis infections during STD screening in this non-traditional venue than during the diagnosis and routine screening that occurred at the clinic. Conversely, a higher percentage of clients, many of whom were symptomatic, tested positive for gonorrhoea and chlamydia during the GMH\&W Clinic than during the Tuesday night outreach. This effort allows us to engage hard-to-reach men who may be among the core transmitters driving the HIV and syphilis epidemics in the DC area. Costs per HIV and syphilis cases detected need to be calculated /estimated for this fruitful outreach endeavour.

\section{5-S4.05 INTRODUCTION OF A SEXUAL HEALTH PRACTICE NURSE INCREASES STI TESTING AMONG MSM IN GENERAL PRACTICE}

doi:10.1136/sextrans-2011-050109.173

${ }^{1} \mathrm{M}$ Y Chen, ${ }^{1} \mathrm{~A}$ F Snow, ${ }^{1} \mathrm{R}$ Cummings, ${ }^{2} \mathrm{~L}$ Owen, ${ }^{3} \mathrm{C}$ El-Hyak, ${ }^{3} \mathrm{M}$ E Hellard, ${ }^{4} \mathrm{~L}$ Vodstrcil, ${ }^{5} \mathrm{C}$ K Fairley, ${ }^{5} \mathrm{M}$ Y Chen. ${ }^{1}$ Melbourne Sexual Health Centre, Melbourne, Australia; ${ }^{2}$ Victorian AIDS Council, Gay Mens Health Centre, Australia; ${ }^{3}$ Burnet Institute, Australia; ${ }^{4}$ University of Melbourne, Australia; ${ }^{5}$ Melbourne Sexual Health Centre, University of Melbourne, Australia

Introduction Increased screening among MSM could improve STI control in this population; however published data on interventions that improve screening rates is limited. The aim of this study was to determine if introducing a sexual health practice nurse (SHPN) into a general practice clinic could increase HIV and STI testing among MSM who attend.

Methods In October 2008, Melbourne Sexual Health Centre introduced a SHPN into a Melbourne general practice with a high caseload of MSM. We undertook an observational study comparing the proportion and STI tests undertaken in the 9 months before (Period 1), and after the SHPN was introduced (Period 2). Consistent with Australian national STI testing guidelines for MSM, complete testing was defined as HIV and syphilis serology, urine test and anal swab for chlamydia, pharyngeal and rectal swabs for gonorrhoea, from the same man on the same date. MSM were stratified and analysed according to HIV status. The Qui-Square Test for Independence was used to compare the difference in proportions of tests. In the case of syphilis tests among HIV positive MSM the median number of tests was compared using the Mann-Whittney U Test. Results Among HIV negative MSM, the propotion of MSM tested, increased from Period 1 to Period 2 as follows; HIV from $57.8 \%$ to $66.2 \%$; syphilis from $59.9 \%$ to $76.6 \%$; urethral chlamydia from $67.7 \%$ to $75.8 \%$; pharyngeal gonorrhoea $62.5 \%$ to $69.9 \%$; and rectal gonorrhoea/chlamydia from $58.5 \%$ to $69.5 \%$ (all $\mathrm{p}<0.001$ ). The proportion of episodes of complete tesing, also increased from $41.1 \%$ to $51.9 \%(\mathrm{p}<0.001)$. Among HIV positive MSM, the proportion of MSM tested, increased from Period 1 to Period 2 as follows; urethral chlamydia from $66.5 \%$ to $80.2 \%$, pharyngeal gonorrhoea $58.7 \%$ to $77.2 \%$ and rectal gonorrhoea/chlamydia from $55.3 \%$ to $75.3 \%$, (all $\mathrm{p}<0.001$ ). Prior to Period 1 , the clinic had a policy of testing HIV positive MSM for syphilis with each three monthly routine HIV monitoring blood tests. Therefore, the median number of syphilis tests was the same in both study periods (median 2, range 0 to 6 , $\mathrm{p}=0.817)$. The proportion of episodes of complete tesing, also increased from $32.3 \%$ to $56.2 \%(\mathrm{p}<0.001)$.

Conclusion The introduction of a SHPN into general practice significantly increased HIV and STI testing among MSM. The magnitude of the effect of this intervention may be greater in MSM high case load general practices where the culture of STI testing is less well established.

\section{5-S4.06 IMPROVING HIV SCREENING AT AN URBAN STD CLINIC}

doi:10.1136/sextrans-2011-050109.174

${ }^{1} \mathrm{~B}$ W Furness, ${ }^{2} \mathrm{~J}$ Coursey, ${ }^{2} \mathrm{~T}$ Flemming, ${ }^{2} \mathrm{P}$ Hess, ${ }^{3} \mathrm{~S}$ Sankar, ${ }^{3} \mathrm{~N}$ Kamanu Elias. ${ }^{1} \mathrm{CDC} /$ NCHHSTP/DSTDP/ESB/FEU, Washington, USA; ${ }^{2}$ CDC/NCHHSTP/DSTDP, Washington, USA; ${ }^{3}$ Department of Health, Washington, District of Columbia, USA

Background At the end of 2006 (the most recent year that data are available), an estimated 1106400 persons in the USA were living with HIV infection, with 21\% undiagnosed. In September 2006, the Centers for Disease Control \& Prevention (CDC) released new 